\title{
La deserción precoz y temprana en el Politécnico Colombiano Jaime Isaza Cadavid de la ciudad de Medellín, una mirada desde los protagonistas y el impacto en su proyecto de vida ${ }^{1}$
}

\author{
Rodrigo J. Carvalho Valencia ${ }^{2}$
}

\begin{abstract}
Resumen
Cada año en Colombia, casi el $48 \%$ de la población universitaria se retira sin terminar la carrera. La tasa más alta lo hace por razones económicas. Otros se retiran porque no comprenden las políticas de la institución, o porque no se sienten a gusto con compañeros y profesores. En el Politécnico Jaime Isaza Cadavid cada semestre, un promedio de mil estudiantes salen de la institución. Muchos de ellos no llenan el formulario de cancelación y se van "sin dejar huella". En las estadísticas del Departamento de Planeación dice que la principal causa es la incompatibilidad laboral, mientras que la causa "otros" llega al $27 \%$.

Esas personas nunca dicen qué pasó o por qué. Esta investigación cualitativa busca encontrar aquellas personas que se retiraron antes de terminar su primer (deserción precoz) o el segundo semestre (deserción temprana); conocer sus sentimientos cuando tomaron esa decisión y cómo afectó sus proyectos de vida y sus vidas laborales, académicas y familiares. Estas son sus historias, las conclusiones y las sugerencias para una política de retención.

Palabras clave: Estudiantes, deserción, universidad, suicidio, decisión, retención, proyecto de vida.

\section{The early attrition and early at the Polytechnic Colombiano Jaime Isaza Cadavid in Medellin, a view from the players and the impact on your life plan}

\begin{abstract}
Each year in Colombia, almost 48\% (MEN, 2010, 3) of the University students dropout from their careers. The highest rate is because economic matters. Others quit because they don't understand the institution politics or because they don't feel comfortable with their mates o teachers. In Politécnico Jaime Isaza Cadavid each semester, an average of one thousand students quit

1 Recibido: 09 de enero del 2012. Aceptado: 27 de febrero del 2012.

2 Rodrigo J. Carvalho Valencia. Comunicador social-Periodista de la Universidad Pontificia Bolivariana. Magister en Educación-Docencia de la Universidad de Manizales. Docente de cátedra del Politécnico Jaime Isaza Cadavid de Medellín y del Tecnológico de Antioquia en las áreas de inglés y Lengua Materna. Correo Electrónico: rodrisopas@hotmail.com
\end{abstract}


from the institution. A lot of them did not fill the cancellation form and, they leave the institution "without trace". The Planning Office says the main cause is work incompatibility (almost $50 \%$ ); while the reason "others" is $27 \%^{3}$. Those people never say what did happen or why. This qualitative research try to find those people who retired before they finished their first semester (sooner dropout) and the second semester (earlier dropout); to know their feelings when they took that choice and how that decision affected their life project and their work, academic and familiar life. These are their stories, the conclusions, and suggestions for a policy of retention.

Keywords: Students, dropout, university, suicide, choice, retention, life project.

\section{Introducción}

Hablar de la deserción estudiantil en Colombia es tocar un tema que afecta a todos: las escuelas, los colegios, las universidades, la sociedad, el gobierno... y a aquellos que se retiran, incluidas sus familias y amistades.

Si bien se ha realizado muchas investigaciones sobre el tema, por lo general éstas se quedan en los datos estadísticos y pocas veces se centran en el alumno, en sus percepciones y sus sentires con relación no sólo a la institución misma, sino con respecto a la carrera o a los profesores o a los compañeros y hasta con su vida misma como futuro profesional y miembro de un grupo social.

Explorar la deserción desde estos aspectos, me ha llevado a ver que las variables cuantitativas se convierten en razones particulares que bien pueden ser toda una historia de vida en la que quedan involucrados no sólo el estudiante; también su familia, sus amigos, compañeros; hasta la sociedad misma.

Cada semestre un promedio de mil estudiantes abandona el Politécnico Jaime Isaza Cadavid de Medellín, ya sea para no volver a estudiar y dedicarse a otras formas de "progresar" en la vida (proyecto de vida); o para irse a otra institución que tiene lo que, a su parecer, el Politécnico no puede brindar.

Motivos para desertar son muchos. Van desde el bajo rendimiento académico hasta la planta física de la institución. Cada una de esas variables tiene raíces profundas en el ambiente familiar y social del estudiante.

Este es el trabajo realizado con estudiantes que en su momento estudiaron en el Politécnico Jaime Isaza Cadavid y que por diversas razones, decidieron suspender sus estudios; ya fuera para continuar en otra institución o para dejar "para más adelante" la continuación de los mismos. Estas son sus percepciones. Lo que sintieron, los que piensan de su futuro y de qué manera esa decisión les ha afectado en su proyecto de vida.

\section{Las invocaciones}

Durante diez años he ejercido como docente de educación superior. En el Politécnico Jaime Isaza Cadavid lo hago desde hace ocho años. También he enseñado en otras instituciones: Instituto Tecnológico Metropolitano (ITM), Universidad Nacional Abierta a Distancia (UNAD), Universidad Minuto de Dios, Universidad Cooperativa de Colombia, Tecnológico de Antioquia y Cedecómputo. Con el tiempo

3 Tomado de la página www.politecnicojic.edu.co Departamento de Planeación. Acerca de la deserción entre los años 2006 y 2010-2. Consultado en Septiembre de 2010. 
he identificado en cada institución un tipo de personaje que llega allí a realizar su carrera. Hasta el punto de tener sus "retratos" (algo así como una tipología) en la se pueden distinguir sus intereses, sus expectativas y sus opiniones en torno, no sólo a la institución donde estudian sino comparándola con otras de la ciudad.

Todo comenzó por un correo electrónico que me llegó acerca de cuántos estudiantes se necesitan para cambiar un bombillo en las universidades de Medellín. Era un correo bastante jocoso en el que, de alguna manera, quienes al leerlo no sólo reían, sino que reconocían las universidades aludidas. Luego, después de revisar más correos del mismo, encontré que en otros países como Costa Rica, Argentina y Chile, también tenían el mismo chiste adaptado a sus propias instituciones de educación superior.

A partir de ese momento quise saber si realmente existe un sujeto en cada universidad; si cada entorno tiene sus sujetos. Las primeras aproximaciones me dieron pistas de que sí. Había que realizar encuestas y establecer parámetros para determinar si en realidad había un tipo de estudiante para cada universidad o si cada universidad requiere un tipo de estudiante.

Hubo un momento en que no supe cómo encontrar ese sujeto particular (el "Homo Politecnicus") sin caer en estereotipos faltos de rigor científico (al menos cualitativo y etnográfico). Así que me incliné por conocer a otro sujeto no menos importante: el desertor del politécnico Jaime Isaza Cadavid. La inquietud comenzó a crecer una vez leí las estadísticas de deserción en el Politécnico que arrojaban un promedio de mil estudiantes por semestre. ¿Por qué se van? Fue la pregunta que surgió ante los números presentados por el departamento de planeación del Politécnico. ${ }^{4}$

4 Politécnico Colombiano Jaime Isaza Cadavid. Oficina Asesora de Planeación. www.politecnicojic.edu.co, consultado en Julio 31 de 2010.
Entonces comencé a preguntar entre algunos ex alumnos qué había pasado y comenzaron las historias de vida. Razones despojadas de datos estadísticos que comenzaron a tener otros matices.

Luego indagué sobre el fenómeno y me encontré con que Spady $(1970,78)$ buscó las raíces de la deserción desde lo individual: "El retiro universitario es una forma menos drástica de rechazar la vida social que el suicidio... Se da como resultado de una falta de interacción con los otros... falta de compatibilidad con el sistema social y el entorno". Vincent Tinto $(1987,12)$ se enfocó en los aspectos académicos: "El estudiante evalúa los beneficios de permanecer en la institución y los costos personales (esfuerzos, dedicación) y si otras actividades son reconocidas como fuentes de mayor beneficio"; Bean $(1980,159)$ destacó los aspectos institucionales: "El estudiante interactúa con la institución, percibiendo lo objetivo: calificaciones, promociones, etc; y también lo subjetivo: como el valor práctico de las materias o de la carrera misma" y Gaviria $(2002,4)$ en lo socioeconómico: "el acceso a un plantel de buena calidad, aparte de incidir de manera notable sobre el rendimiento académico, es un privilegio casi exclusivo de quienes tienen dinero y pueden comprarlo. En otras palabras, los pobres raramente pueden comprar una buena educación para sus hijos, lo que contribuye a concentrar las oportunidades y a retardar la movilidad social".

Tinto, un investigador norteamericano, quien lleva más de 50 años estudiando el fenómeno y sobre el que ha formulado algunas teorías, destaca que los procesos de acomodamiento de los estudiantes con relación a su paso por la universidad pueden influenciar en sus decisiones de si continuar en el claustro o, como sugiere Durkheim (1899, 136), "...no se adelanta cuando no se marcha hacia algún fin... perseguir un fin inaccesible es condenarse a un perpetuo estado de descontento". Esto invita al estudiante a cometer un suicidio académico porque las circunstancias 
lo "acorralan"; por lo tanto, "suicidarse" (en nuestro caso, desertar) es una forma de abandono de las situaciones que incomodan a la persona que lo comete.

Más adelante, en la "Era del Vacío", Lipovetsky $(1986,65)$, plantea un panorama más desolador: "La deserción como una forma de suicidio de una sociedad que lo tiene todo y ya no le importa porque lo que realmente importa es el instante, algo así como una falta de compromiso de las personas consigo mismas y con la sociedad, la que ya con sus reglas y objetivos no es más el sueño dorado de estudiantes y profesionales".

Vienen, luego los estudios cuantitativos sobre la deserción. La mayoría de las universidades colombianas vienen estudiando el fenómeno que ha tenido sus altibajos a lo largo de la historia, pero que en los últimos años se ha hecho más notorio porque afecta, desde los planes gubernamentales de ofrecer educación para todos; se afecta la comunidad (¿la sociedad?): otra persona más en las calles; se afecta el grupo familiar: es una carga económica más para la familia; hasta aspectos económicos para la institución (solo con los mil estudiantes que se retiran por semestre del Politécnico Jaime Isaza Cadavid, la cifra que se deja de percibir supera los 700 millones de pesos, eso sin contar los gastos que cada estudiante genera), además que con el retiro del estudiante, otros estudiantes que sí querían estudiar no lo lograron; para los docentes puede resultar perjudicial porque disminuyen las posibilidades de nuevos empleos.

A comienzos de 2010, el Ministerio de Educación Nacional emitió su boletín número 14 en el que manifiesta la preocupación nacional por el fenómeno de la deserción (48\% de los estudiantes universitarios se retira antes de terminar la carrera).

En 1996 la Universidad de Los Andes de Bogotá creó una base de datos nacional para detectar el fenómeno con las universidades del país llamado SPADIES
(Sistema para la prevención y análisis de la deserción en las instituciones de educación superior). Hasta el momento hay 238 instituciones adscritas al sistema. Con base en estos datos, el Ministerio de Educación ha propuesto programas de retención que incluyen asesorías psicotécnicas para bachilleres, préstamos blandos y, en combinación con la empresa privada, préstamos condonables y becas. A su vez, las universidades han propuesto estrategias de retención con el objeto de disminuir las cifras de deserción. En algunos (como en el Instituto Tecnológico Metropolitano, se optó por hacer seguimiento de los estudiantes con las familias de éstos para saber de su comportamiento)

En Medellín la Universidad Nacional (2005), Politécnico Jaime Isaza Cadavid (2008), EAFIT (2008), la Universidad de Antioquia (2005) y la Universidad de Medellín (2007). En Bogotá están las investigaciones de Universidad de La Sabana (2004), Los Andes (2007) y Universidad del Rosario (2007); también hay otra de la Universidad de Cartagena (2007) en Bolívar y otra de la Universidad de Los Llanos (2006)

El promedio de deserción en estas instituciones varía entre el 15 y el $18 \%$. Cada una hizo un rediseño de las políticas de retención que incluyen facilidades de pagos en aquellas cuyo motivo principal de retiro fue el económico; mejoras en la atención para los universitarios, capacitaciones para "asumir" la universidad; facilidades con Bienestar universitario para disminuir el costo de los pasajes (convenios con las transportadoras); algunas instituciones, como el Politécnico, implementó darles el almuerzo a aquellos estudiantes con menos recursos.

En 2005 la facultad de ingenierías de la Universidad de Antioquia realizó una investigación para responder al interrogante del número de deserciones en el claustro y descubrió, que gran parte de éstas eran de índole académica, pero con mayor énfasis en lo interinstitucional; 
esto es, que salieron de la Universidad para irse a otra institución (59.6\%); sólo el $7 \%$ adujeron problemas económicos. Ese porcentaje de deserción interinstitucional se debe, más que todo, a problemas de orden público que afronta constantemente la universidad. El promedio de duración de una carrera es de 6 años, incluido el tiempo que se "pierde" con los paros y las protestas.

El Instituto Tecnológico Metropolitano de Medellín (2006) realizó su estudio sobre la deserción. El resultado fue de un 17,3\% (Alerta Estadística, 2007) ${ }^{5}$ durante el segundo semestre de 2006. Se había incrementado la deserción con relación al semestre anterior, que fue del $15,8 \%$. Sin embargo, en una nota aparecida en el Tiempo, para 2008 índice fue de 11\%, después de implementarse algunos programas de retención entre los que se incluye una política de alivio en las matrículas y facilidades de pago semestral. ${ }^{6}$

La Escuela de Administración y Fianzas - EAFIT (Montes Gutiérrez, 2010) realizó una investigación cuantitativa en torno al tema y se determinó que, en su orden, los motivos de retiro principales fueron en su orden: la elección errónea de la carrera, el rendimiento académico, el factor económico y el costo de oportunidad por trabajar y emprender un negocio (incompatibilidad horaria).

La Universidad Nacional publicó una investigación sobre la deserción en el Politécnico Jaime Isaza Cadavid entre los años 2000 y 2006 (Maya Guerra, 2008, 57-58) en donde destaca:

"De acuerdo con los resultados un $25 \%$ de los encuestados reportaron razones de tipo financiero para su retiro y un $29 \%$ reportaron razones laborales, mientras que un $25 \%$ expusieron razones estrictamente

5 Oficina de Planeación. Alerta Estadística. Año 4, № 13, Mayo 2007.

6 El Tiempo. Sección: Nación. Noviembre 28 de 2009. laborales y un $19.5 \%$ razones estrictamente financieras. Un $48 \%$ no tuvieron razones ni financieras ni laborales. Por otra parte un $11.5 \%$ argumentaron razones institucionales $y$ un $19.5 \%$ por razones académicas. De estos un $6.5 \%$ lo hicieron por razones estrictamente institucionales y un $12 \%$ por razones estrictamente académicas. Un $72.5 \%$ no tuvieron razones ni institucionales ni académicas".

Después me di cuenta que los autores y las investigaciones tenían como objeto estudiar el fenómeno y sus resultados (investigación cuantitativa) y que pocos (o ninguno) se han preocupado por escuchar las voces, a no ser por los formularios que se deben llenar a la hora de cancelar un semestre; pero, ¿Y esos que nunca llenaron formularios y que "desaparecieron" de las aulas de clase sin un adiós, sin una razón. Estos son los que Rivera (1996) califica de "desertores precoces."

Según las estadísticas la mayoría de las deserciones ocurren en los primeros tres semestres. Esto se debe, más que todo, a que el sujeto no tiene muy claro cuál es el objetivo de su ingreso a la universidad. Para algunos es una alternativa mejor que quedarse en casa esperando el ingreso a otra institución. Para otros es ir a "ver" si se ajusta a la expectativa.

La mayoría de las investigaciones apuntan a que el estudiante recién egresado del colegio no fue capacitado para enfrentar un cambio en la disciplina y en los estilos de estudiar, de calificar, de relacionarse con sus pares.

Aunque los retiros académicos ocurren también en los semestres superiores, las causas de éstos van más ligadas a variables de tipo laboral (cambios de horarios o traslados) o económico; pocas veces tiene que ver con lo académico.

El retiro académico (que puede ser deserción o mortandad académica) conlleva unos efectos negativos para el estudiante, para su entorno, para sus 
relaciones sociales y para la universidad misma. Y siguiendo con los enunciados de Durkheim (1899) sobre la anomia y el suicidio se vislumbra un panorama triste para el estudiante que se retira; sin embargo algunas de las entrevistas plantean casos contrarios: se retira porque no era capaz, porque fue un elección errónea, porque hay que esperar a tener un poco más de madurez para enfrentar esa responsabilidad.

Lo que pretendía la investigación era mostrar las historias de aquellos que vivieron la deserción en carne propia, escuchar sus opiniones en torno al fenómeno, si realmente se sienten menospreciados por la sociedad, o simplemente consideran que su decisión fue un "alto en el camino" para esperar mejores oportunidades, sean éstas de carácter económico o académico.

Al buscar, entre las expresiones de esos estudiantes, cómo cambió (o si no cambió) su "proyecto de vida" cuando decidieron que no seguían "luchando" contra un montón de "problemas" que pasan por los de carácter académico, laboral, económico, encontré voces que hablaban de sus inconvenientes, de sus visiones.

Algunos entrevistados estuvieron en clases en el Politécnico porque no querían quedarse un "semestre vagando" o "mientras espero pasar a la de Antioquia y ésta es la más barata de todas". Hay quienes apuntan a considerar que la universidad no tiene nada qué enseñarles salvo la "legalización" de un título que les dé "estatus" profesional; porque lo demás ya lo saben.

Sin embargo, como dice Bourdieau (1991, 49), a veces "las expectativas de los estudiantes pueden estar matizadas por otros referentes más ocultos que tienen que ver con sus entornos sociales, familiares y sociológicos". Así que se hace necesario buscar interpretaciones a esas respuestas "inconscientes" que lo que hacen es reflejar formas de asumir la vida que provienen de los primeros estadios vitales en el seno de la familia o en la escuela.

\section{Las gramáticas...}

En la medida que me adentraba en las entrevistas pude encontrar otras opiniones, otras visiones acerca del tema. Para alguno fue simplemente un cambio de lugar de estudio. El Politécnico no colmaba las expectativas, o en otro caso: "Yo no esperaba quedarme allí, era nada más para tener qué hacer mientras pasaba a la de Antioquia".

A partir del proceso de escucha e interpretación de las entrevistas (un total de 20 realizadas al azar) practicadas con estudiantes que se retiraron antes de finalizar el primer semestre (deserción precoz) se trata de "leer" sus respuestas, mirar sus vidas para tratar de comprender la razón de sus decisiones que, como ya se dijo, pueden no ser de carácter negativo; que bien pudieran ser de carácter práctico (toda vez que las decisiones tomadas podrían resultar beneficiosas a mediano o a largo plazo).

El propósito fue describir situaciones y eventos relacionados con la deserción en el Politécnico Jaime Isaza Cadavid de la ciudad de Medellín. Esto es, decir cómo es y cómo se manifiesta determinado fenómeno. Los estudios descriptivos buscan especificar las propiedades importantes de personas, grupos, comunidades o cualquier otro fenómeno que sea sometido a análisis. Evalúan diversos aspectos, dimensiones o componentes del fenómeno o fenómenos a investigar. Esto es, en un estudio descriptivo se seleccionan entrevistas de profundidad y con base en las respuestas se hace una panorámica en la que lo importante son las voces de aquellos que resolvieron salir de la universidad aun sin terminar sus estudios; o, como en los que salen antes incluso de iniciar el semestre, para que cuenten qué ocurrió en realidad y no como lo plantea el dato estadístico. 
La parte más difícil fue hallar estudiantes retirados. Hubo que buscarlos a partir de los relatos de otros o de las faltas consignadas en las planillas de asistencia; luego contactarlos y establecer las pautas para una entrevista a profundidad, a partir de un modelo de entrevista en el que se exploraban los datos personales y sociales relevantes.

Hablar de fracasos es aceptar que se hizo algo de manera equivocada. En este sentido algunos de los entrevistados manifestaron no haber perdido nada con salir de la carrera. En el caso de quienes estuvieron en el Politécnico a manera de "escampadero" eso fue todo. Un lugar que les permitió enfrentar de una manera menos estresante el proceso universitario, para luego retomarlo con ahínco en otra institución.

En el hogar los padres sueñan con el profesionalismo de sus hijos (que sea lo que yo no fui) y porque, según los cánones, ser profesional en este mundo competitivo es la mejor manera de tener "con qué" o de tener un futuro. Futuro que nadie dice qué es exactamente, lo mismo con el "tener con qué" (se habla de la inexactitud del mensaje -un mensaje poco claro-), se genera el miedo a equivocarse, miedo al fracaso.

\section{Las voces..}

\section{Fran, la esperanza de un cambio para continuar}

"Yo entré a esa carrera porque me gusta el fútbol y lo juego bien. En el barrio donde vivo puedo darles clase a los muchachos; pero el municipio me dice que debo tener una profesión (al menos una tecnología) para que me contraten como entrenador de niños. El problema es que no me la llevé bien con los profesores. Mire, en el primer semestre tuve problemas con Lengua Materna y con Matemáticas. Fuera de eso también había una materia llamada Humanidades. El profe hacía leer muchos documentos y a mí me quedaba difícil leerlos y hacer los informes. Perdí tres materias de las seis materias con que comencé.

A mí me gusta estudiar; pero creo que los profesores no me tienen paciencia. Mire, hubo uno que me tildó de retrasado y eso me dio mucha 'piedra' por eso me le fui de la clase. Soy tan de malas que al semestre siguiente me tocó el mismo. Me tuve que salir.

Yo sé, profe, que en cualquier momento regreso. Ahora estoy consiguiendo la plata de la matricula; aunque me da mucha "piedra" entrar de nuevo porque mis compañeros están muy adelante"

"No lo miran a uno, profe; especialmente los de matemáticas, llegan, llenan el tablero de fórmulas, hacen un ejercicio y nos dejan en el salón resolviendo"

\section{Yadira: El futuro está en otras latitudes}

"En mi pueblo, Quibdó, todos me decían que debía ampliar los horizontes. Chocó en un buen departamento, pero no hay futuro. Así que decidí venirme para Medellín y el mejor lugar era el "Poli" por la carrera de deportes. Además porque para los deportistas de alto rendimiento dan beca. Así que ingresé a Licenciatura en Deportes. De inmediato me dieron espacio en la selección de la universidad, una de las mejores a nivel nacional. El problema está que no puedo dejar el deporte de lado, pues son varios los compromisos deportivos a nivel internacional.

No me siento mal por dejar el Poli; al contrario, siento que mejora mi situación. Esa universidad me ofrece, no sólo estudio y la posibilidad 
de jugar a nivel profesional, también me ofrece un salario y la expectativa de permanecer en ese país con mi familia".

"A mí me gusta el Poli, es una universidad buena; el decano fue muy generoso al permitirme ingresar a la selección. Sé que habló con varios profesores para que me hicieran los exámenes o talleres cuando tuviera que ausentarme... Pero, mire, en Méjico me ofrecen de todo. Yo no sé como sea allá, pero me dicen que si juego bien y me comporto, las cosas pueden ser muy buenas."

\section{Luisa: Mientras esperaba pasar a la de Antioquia}

"Tengo tres hermanos, todos ellos estudiantes universitarios. Mi mamá tiene una tecnología que no ejerce, y mi papá también es profesional en Administración, tiene mediana empresa. Así que siempre me ha gustado estudiar. Como en la casa cada uno tiene libertad para elegir, a mí siempre me gustó la Química, mis profes en el colegio me impulsaron a elegir esa carrera porque me iba bien en el laboratorio".

"Hice el examen tres veces. Usted viera, profe, la primera vez no alcancé el puntaje de corte que para las ingenierías es de 68.300 puntos. No se imagina la sensación de fracaso, pues tenía a la mano exámenes de la de Antioquia, los hice y me iba bien. ${ }^{7}$ Entretanto ya había solicitado el ingreso al Poli y había pasado la entrevista; así que me matriculé. La segunda vez estuve a menos 50 puntos del corte; pero no quise abandonar. Ya la tercera vez pude

7 En la universidad de Antioquia los exámenes de admisión se los quedan los estudiantes pues cada semestre varían y existen hasta tres o cuatro versiones, por lo que se convierten en documentos de estudio para los aspirantes. Esto no ocurre con la Universidad nacional. lograr el corte. Entonces me matriculé. En 2010-2 me matriculé a la de Antioquia y seguí en el Poli; pero no aguanté el ritmo. Dos carreras se hace muy difícil; más aún cuando una cree que las materias son iguales pero no, la exigencia en la de Antioquia era más fuerte (bueno aquí también), así que apenas aguanté dos meses en el segundo semestre. Me tocó retirarme.

"Lo bueno es que en esos dos semestres aprendí mucho; aunque de las materias del segundo semestre apenas me reconocieron dos. Además conocí buenos compañeros.

"Yo me metí a la Tecnología en el horario nocturno; así que pude compartir con gente mayor que yo (era la niña del salón) y que de verdad me colaboró mucho en las materias más difíciles

"En mi casa, nadie me dijo nada. Mi papá pagó con gusto el semestre en el Poli porque consideraba que era una buena manera de "adelantar" materias con relación a la carrera”.

\section{Johanna: Es mejor en la}

Nacional... y más barato.

"No me sentía bien en el Poli. Me gustaban mis compañeros; pero sentía que no era lo mío. Además los gastos. No es lo mismo pagar setecientos mil pesos por semestre que pagar ciento ochenta en la Nacional; además, la calidad de la educación es diferente. Usted me perdona, pero en la Nacho ${ }^{8}$ el estudio es mucho más comprometido.

"No es que en el Poli las carreras sean malas, pero una se da cuenta que los profesores en la Nacho son más exigentes. Allá sí que hay que

8 Los estudiantes de la Universidad Nacional de Colombia-sede Medellín, la reconocen por "La Nacho". 
"mazear" mas, de lo contrario rapidito lo sacan de la U.

"Al principio me sentí rara en la Nacional. No sé por qué, pero los estudiantes son más comprometidos con el proceso. A veces siento que no tengo ni tiempo para recrearme. La función es estudiar para sacar adelante las materias. Además siempre existe la posibilidad de obtener una beca de honor si el promedio supera el 4,5 y eso hay que intentarlo, pues la matrícula queda en mil pesos por semestre. En el Poli no hay esa política. Becas son para los deportistas de alto rendimiento y que demuestren medallas en torneos nacionales o internacionales".

\section{Javier: simplemente,}

\section{no se puede...}

“Me dediqué a 'gozarme' el semestre. Todo parecía muy fácil. Me junté con un grupo de compañeros y nos dedicamos a 'rumbear', a pasarla bien en el Poli, íbamos a piscina, jugábamos fútbol. A veces nos quedábamos en las escalas del Metro tomando cerveza. Al fin y al cabo allá no llaman a lista así que no hay peligro de cancelar por faltas de asistencia. Lo malo es que los profesores hacen sus talleres y yo no caí en cuenta de pedir que me 'llevaran'en algunos de ellos. Finalizando el semestre tuve que cancelar tres materias que las tenía muy perdido y traté de mejorar en las que me parecían más fáciles: Lengua Materna y Humanidades; pero no pude lograr el promedio. Perdí el semestre.

Ahora estoy aquí en este supermercado. Trabajo todo el día. Varios de mis compañeros de trabajo también estudian en otras universidades y me han hablado para que regrese; pero, la verdad, profe, no tengo ganas. Al menos no todavía... hasta que me responsabilice. Perder el semestre también hizo que en mi casa no me dieran más nada para mí. El "cucho" me dijo que si quería estar en la casa debía ponerme a trabajar.

El trabajo en "La Vaquita" (el nombre del supermercado) es bastante agotador: uno arranca a las siete de la mañana y está saliendo a las ocho de la noche. Eso de lunes a sábado.

Me toca de empacador y a veces, me toca llevar los mercados a domicilio. Uno se gana sus pesos, pero no es suficiente para pensar en universidad. Una vez le dije al patrón y me dijo que sí, que estudiara nocturna; pero ahí me di cuenta que eso es bien difícil. Si así salgo bien cansado, cómo será estudiando de noche. ¿A qué horas descansa uno?.

"La verdad es que me gustaría estudiar. Quisiera volver al Poli, pero no hay la plata y, la verdad, me da verraquera regresar y ver a mis compañeros ya bien adelantados $y$ uno apenas comenzando de nuevo. De pronto me meto a hacer una tecnología en otra institución, tal vez el ITM". 9

\section{Edwin: No era mi carrera...}

"Los profesores no sabían explicar. Perdí Matemáticas 1. El profesor entraba, escribía unas fórmulas en el tablero y se iba. Al final perdí y eso me rebajó mucho el promedio. Al siguiente semestre las mismas Matemáticas cambiaron: ya estaban enfocadas al tema de Contabilidad, así que aunque me tocó repetir fue como volver a verla por primera vez. La perdí de nuevo. Al verla por tercera, la materia estaba enfocada a ingenieros, así que era más especializada. Por poco la pierdo. Por eso, mejor me retiré. Este semestre

9 Instituto Tecnológico Metropolitano. Una institución universitaria adscrita al Municipio de Medellín. Según los estudiantes, una de las más baratas de la ciudad. 
pasé a la Universidad de Medellín. Me inscribí a Contaduría. No quise cambiarme de carrera en el Poli porque no me parece que sea buena opción. Mis amigos dicen que es mejor la Medellín por los profesores; aunque algunos de ellos enseñan en el Poli, no sé por qué no son mejores allá que en la Medellín. LO otro es que con Ingeniería de Calidad tenía que relacionarme con la gente y a mí me gusta más la idea de tener mis empleados y no tener que relacionarme con ellos".

"No me interesó hablar con nadie, pues no vi razón para quedarme; además, con haber obtenido la beca del Fondo EPM, pues lo mejor era buscar ingreso en la mejor universidad. En este caso la de Medellín. Mis compañeros y amigos dicen que tengo más posibilidades de conseguir un buen empleo si me acredito como egresado de allí. Claro que el "Poli" es bueno, pero la Medellín tiene mayor reconocimiento en el medio y con relación a esta carrera (Contaduría) y en Derecho. En cambio la carrera del Poli no es tan buena no están bien mirada... Y es que en realidad muchos compañeros se sienten un poco defraudados porque las materias no colman sus expectativas".

\section{Ana María, No era lo que anunciaban...}

"Siempre soñé con la actividades aéreas y pensé que esta carrera me abriría las puertas para ser, al menos, auxiliar de vuelo y conocer muchos lugares. Mi pasión es volar. Pero al llegar a la universidad encontré que la carrera es para administración de terminales aéreos y me parece que no hay muchos lugares de esos para trabajar.

"Me di cuenta que en realidad esa carrera es como una carrera normal de administración y eso de volar es mejor hacer la carrera de Auxiliar de vuelo. Lo que ocurre es que esa carrera es más costosa y no hay tanto dinero para pagarla. Yo creí que en Poli lo podría conseguir, pero me equivoqué.

"Ahora estoy pensando en recoger el dinero para meterme a la academia de Aviación."

\section{Alejandra: Primero el trabajo...}

"A mí me gusta el estudio, pero ya ve. No se puede, más cuando una tiene que estar al frente de la empresa. Mi papá tiene una empresa de confecciones y yo soy la que hace todo allá. Así que no pude acomodar las materias que me asignaron, porque a veces, especialmente en las noches, tenía que trabajar y no podía atender las clases.

"No me siento mal por no poder seguir estudiando, pienso que tal vez mas adelante me meta a terminar la carrera; pero, vea, cada día que pasa pienso que esa carrera no me va a servir para mi futuro.

Es decir, no es que no sirva, es que por ahora debo sacar adelante la empresa familiar. Mi papa quisiera, pero dígame, ¿Quién se va a poner al frente?; por ahora soy yo. Me da susto saber que me voy poniendo vieja para reiniciar la carrera; pero tengo confianza que en el momento que reinicie sí voy a terminarla. $Y$ sé que de pronto tenga manera de ejercerla."

\section{Juan Fernando ¿Para qué esas materias...?}

"Al principio me gustó la idea de ser "agropecuario", me soñaba con los conocimientos propios para mejorar el ganado de la finca. Tenía facilidad para practicar lo que aprendiera en el Poli. Era una ventaja.

"La cosa cambió cuando vi las materias del primer semestre. Yo 
pensaba que al ser una tecnología uno arrancaba de una vez a "ver" materias relacionadas con la carrera, en mi caso, con el ganado. Pero no. Matemáticas, Química, Humanidades, Geometría, y en casi todas, nada que ver con ganado o por lo menos aplicadas a la agropecuaria.

Pero mi peor relación fue con esas materias Química y Matemáticas. Por ellas no seguí en el Poli... No sé, tal vez era que los profesores no llegaban con su discurso. A veces veía que la profe de Química era muy 'cerrada' en sus conceptos, muy escuelera...

"El de matemáticas es que llegaba, escribía un ejercicio en el tablero y se iba. Muy pocas veces lográbamos hablar con él.

"Había otro problema: Yo trabajo, profe, porque así me sostengo. Pero el problema es que en el primer semestre el sistema del Poli asigna las materias no según la necesidad sino como salga. Así que me tocaba ver materias en la noche, en la mañana, al mediodía, tuve una a las dos de la tarde. Tuve que hacer muchas 'marañas' para poder asistir a las materias y también para hacer mi trabajo, que también es de horarios extendidos.

"Entonces me salí. No quise seguir. No sé, profe; es que de pronto la edad no contribuye y me parecía que ese primer semestre era algo así como "perder un tiempo precioso" con esas materias de "relleno".

"Así que mejor me fui para la Universidad Minuto de Dios a estudiar Trabajo Social. No es ni parecido a Agropecuaria; pero, la verdad, es lo que me gusta. Incluso, me encanta la idea de trabajar en un proyecto social que se viene gestando en mi pueblo (Ituango) y ya lo tengo hablado hasta con el alcalde.

"No siento haber perdido nada al salirme del Poli, al contrario hay co- sas que agradezco haber aprendido allá. Lengua Materna fue una de esas cosas. Tanto que al presentar el "pensum" inmediatamente me validaron la materia allá en la Uniminuto”.

\section{Las esperanzas...}

Al final, las conversaciones con desertores apuntan a unas cuantas sugerencias para hacer más esperanzadora la educación en el Politécnico:

\section{Lo económico}

Estudiar se convirtió en una exigencia de la vida para "salir adelante", por eso no se concibe una persona sin estudios profesionales; en el caso de los docentes, una maestría. El problema se agudiza con las universidades; pues las públicas tienen cada vez más problemas y menos cupos y aunque el número de universidades privadas aumenta, los costos son cada vez más altos.

Hay que tener en cuenta que el gobierno ha provisto mecanismos para realizar préstamos blandos a los estudiantes de menos recursos; así mismo las universidades han optado por partir las matrículas de manera que se haga menos oneroso "sacar" de una vez el costo de un semestre.

En el Politécnico se ha propuesto el pago del semestre por cuotas, para garantizar la permanencia del estudiante que sale por motivos económicos (mayor facilidad de pago).

\section{Posibilidad de hacer el propio horario}

Los estudiantes que ingresan al Politécnico Jaime Isaza Cadavid deben ajustarse a los horarios que adjudica el sistema. De ahí que muchos retiros se dan porque esos horarios resultan incompatibles con los horarios laborales.

Si desde el principio, estudiantes con necesidades laborales pudieran establecer unos horarios compatibles con los horarios de trabajo, quizás la deserción 
podría disminuir en algo. Así, el estudiante es libre de escoger los horarios y las materias que quiere estudiar. Es decir, si quiere ver sólo una materia en determinado horario, la paga y ya. Es cosa del propio estudiante decidir cuánto se va a tardar en terminar la carrera.

\section{Materias básicas más enfocadas a la carrera}

Dicen los estudiantes que hay una diferencia profunda en las maneras de enseñar de cada docente (en el Politécnico Jaime Isaza Cadavid existe lo que se llama "libertad de cátedra"); en el caso de Lengua Materna, mientras unos docentes "enseñan" ortografía, otros se concentran en las competencias básicas (saber leer, escribir y hablar en público). Hay docentes de Matemáticas que enseñan los conceptos básicos, otros simplemente enseñan lo que saben de matemáticas puras.

Las humanidades, dicen, son filosofía aplicada a la vida. Sólo que los estudiantes no "quieren" leer, o hacer lecturas críticas de la realidad (dicen docentes) sino que se enfoquen en la carrera.

Hay estudiantes que dicen que una materia como "Introducción al área" debería estar enfocada a mostrar los fundamentos de la carrera; sin embargo se queda en mostrar los aspectos misionales y fundamentales de la universidad (manual de convivencia, estudiantil y docente).

Jaime Garzón (1997) hacía el apunte en que "Lo que enseñan en la universidad difiere de lo que uno necesita"10. Kaplún (2004), afirma lo mismo cuando solicita "indisciplinar" la universidad y mira la enseñanza con estas palabras:

"Una primera cuestión es que, en principio, se enseña lo que se sabe. Esto plantea un primer problema: Io que se sabe y se enseña suele ser

10 Esta afirmación la hizo durante su presentación en la Corporación Universitaria Autónoma de Occidente, Cali, Febrero de 1997. Video bajado de www.youtube.com. mucho más el producto de textos $y$ enseñanzas recibidas de otros que de la producción propia de conocimientos... Chevalard (1997) ha descrito como la diferencia que se produce entre el saber académico y el saber didactizado, el saber que se enseña. Este último es siempre diferente que el saber académico, en tanto está sujeto a opciones didácticas y condicionantes institucionales: qué se dice y qué no, en qué orden se presenta, son decisiones que toma el docente y/o la institución, a través de la construcción de los currículos y las opciones metodológicas. Pero cuando estos docentes e instituciones tienen una relación muy indirecta con el conocimiento académico y no participan ellos mismos en su creación, esta distancia suele acentuarse.

Hay entonces un riesgo mayor de que los conocimientos sean presentados de un modo ahistórico y acrítico, sin una adecuada ubicación geopolítica, sin referencia a los contextos en que fueron producidos, las necesidades que buscaban satisfacer, los problemas que querían resolver. Fácilmente se aceptará a los "autores conocidos" como los únicos existentes o, más frecuentemente, se usará la versión predidactizada de los manuales para enseñarlos. En cualquier caso tanto docentes como estudiantes se asoman muy escasamente a los procesos de investigación, que aparecen como una caja negra de la que ya salió un conocimiento verdadero y autosuficiente..."

Un caso que "asusta" a los estudiantes es el inglés. Como el Ministerio de Educación Nacional impuso del modelo del Marco Común Europeo, en el país se tomó al pie de la letra. Ahora es necesario que un estudiante, que ha visto el Inglés como una imposición, gracias a docentes que insisten en hablar todo el tiempo y donde la gramática se volvió el "fucú" entonces 
los estudiantes escuchan sin entender. Al cabo de un tiempo prefieren cancelar la materia o perder el semestre.

Habría que buscar docentes que en lugar de enseñar busquen mecanismos para "enamorar" estudiantes a la materia. Lo demás podría ser más fácil para el aprendizaje de una lengua extranjera.

\section{Profesores más humanos}

El 90\% de los docentes del Politécnico Jaime Isaza Cadavid son de cátedra. La gran mayoría labora por lo menos en otra $\mathrm{u}$ otras instituciones. Generalmente las clases (de 90 minutos) terminan y el docente escasamente tiene tiempo de hablar con sus pupilos y pocas veces atienden quejas, reclamos o sugerencias.

Al ser los más cercanos de los estudiantes deberían tener un poco de tiempo para escuchar sus inquietudes. Es posible que un minuto o dos de escucha puedan hacer que un estudiante se "enfoque" en los estudios y no deserte. ¿Cuántas deserciones pudieron evitarse con un docente escuchando las quejas de los estudiantes?

El departamento de Psicología del Politécnico sugiere a los docentes enviar a los estudiantes que muestran señales de depresión, o que cuando un estudiante pierde por tercera vez una materia sean los profesores, junto con los estudiantes, quienes diseñen una metodología para enfrentar la materia. Ante esto hay docentes que afirman no tener tiempo ni de diseñar estrategias ni hacer acompañamientos, pues esto no es de su competencia-además ese tiempo no lo pagan-, dicen.

\section{Por una educación}

\section{acorde con la vida}

Algunos alumnos consideran que hay materias en sus carreras que desconocen los adelantos de la tecnología. Profesores que no conocen las TIC (Tecnologías de la Información y la Comunicación), por lo tanto, en ocasiones no admiten este tipo de tecnologías en sus clases. Es como si no se admitiera que los aprendizajes de los estudiantes cambiaron. Ahora se habla de "jaulas" y no de aulas clase. Se habla de una inserción de las nuevas tecnologías ya no como un "demonio" que todo lo acaba sino como herramientas, que bien utilizadas pueden dar excelentes resultados a la hora del aprendizaje y el desempeño.

Esto incluye las lecturas que hacen los jóvenes. Es normal escuchar en los pasillos de la universidad a los docentes decir que "estos muchachos no leen". Hay que desglosar qué es "no leer" para los docentes y para los estudiantes. Ya cambió el término porque los jóvenes pueden hacer varias lecturas: lo oral, lo visual, lo escrito y lo auditivo.

Entre tantas posibilidades para mejorar el tema de la deserción en el Politécnico tenemos:

$>$ Son numerosas las voces que hablan de una universidad "humana" y moderna. La que actualmente tenemos se la considera la universidad del siglo XVII pensada para obreros y empresas que hoy, ha cambiado con los nuevos paradigmas económicos. Se habla, entonces de universidad para la creación de empresas y no de empleos. Universidades para las condiciones globalizantes del siglo $\mathrm{XXI}$ que busca profesionales con conocimientos de idiomas, de sistemas y con capacidad de resolver problemas inmediatos.

> Pero, a la vez, universidades que hagan leer a sus estudiantes, a interesarse por el otro, a comunicarse. Hay quien dice que a pesar de todos los adelantes tecnológicos, del incremento de redes sociales, de tener a la mano celulares, portátiles y demás artilugios para la comunicación, cada vez nos comunicamos menos.

> Hay que buscar mecanismos de interpretación (lecturas profundas) para desentrañar la complejidad del ser humano. Se habla de nuevas materias 
en las que se cuentan "tolerancia", "resolución de conflictos"...

$>$ Sería necesario que las universidades "escucharan" esas voces". O que los docentes, en su rol de principales interlocutores con los estudiantes fueran mediadores en las decisiones de esos estudiantes que quieren retirarse.

> La Universidad debería realizar mejores políticas de selección de estudiantes. Por el momento hay una selección basada en entrevistas que a veces no son acordes a los perfiles de alumnos que ingresan. Esto produce deserciones cuando el estudiante que se inscribió a una carrera encuentra que no era lo que quería. Esto implica una implementación de los métodos de inducción, sea antes del inicio de clases o durante las primeras semanas de clases para que los estudiantes tengan clara idea de lo que van a hacer en su vida como estudiantes del Politécnico.

> Debería haber una integración con los colegios de la ciudad a través de "bazares" académicos y en los que las universidades no sólo exhiban sus carreras sino perfilar a los aspirantes mediante pruebas sicotécnicas.

> La universidad debería establecer un perfil de cuál es el tipo de estudiante que quiere el Politécnico Jaime Isaza Cadavid (el sujeto politecniano); de esa manera, se podría evitar un buen porcentaje de deserciones. Para ello deberá consolidar programas que le den sentido a dicho perfil. Es decir, materias bien diseñadas, docentes que no sólo impartan la materia; también consolidar los grupos de investigación en los campos de Administración, Deportes y Agropecuaria que son los que más "acogida" tienen en la institución.

> Implementar o mejorar los mecanismos de detección temprana. Esto se podría lograr si los docentes prestaran mayor atención a sus estudiantes, en lugar de ser unos "maestros taxistas" que vienen, dictan su clase y se van. Lo que el alumno piense o diga en torno a los programas o las materias se tienen en cuenta de manera anecdótica. La institución tiene un instrumento de evaluación estudiantil al desempeño docente; en alguno de sus ítems se pregunta por la percepción personal en torno al docente y a la materia. En ocasiones se escucha a los estudiantes quejarse de tal o cual profesor, sin embargo, a pesar de las quejas, pocas veces se hace algo por cambiar las situaciones. Actualmente (Septiembre de 2011) los estudiantes vienen realizando una serie de asambleas en las que no sólo se discute la Ley 30 sino también una serie de políticas de la universidad con relación a la atención de estudiantes. Como un grupo de retiros se refiere a estudiantes de la nocturna, hay quejas que hablan de que no se prestan servicios administrativos, de salud y de asesorías a partir de las 5 y 30 de la tarde.

$>$ Las quejas de los estudiante en torno a docentes que no escuchan, que no atienden quejas o que no siempre son los mejores calificados (según ellos) se quedan en las anécdotas porque, dicen, nunca se les presta atención a los reclamos. Ante estos hechos las evaluaciones docentes nunca cumplen el cometido de determinar que un docente mal calificado deba ser retirado de la institución. Se propone crear o designar mentores, docentes que no sólo enseñen sino que escuchen y que sirvan de puente entre el alumno y la administración para tomar decisiones.

$>$ En cuanto a la pertinencia de las materias, la institución viene trabajando en la adaptación de programas con las TICs para hacer que el estudiante vaya adquiriendo la cultura del aprendizaje virtual. Para ello no sólo revisa programas sino que busca docentes 
que comprendan el significado de lo virtual, lo entiendan y lo apliquen con sus estudiantes.

$>$ Una queja generalizada (entre desertores y no desertores) es la de falta de implementos que enlacen el aprendizaje en el aula y la vida productiva. Se habla de recursos tecnológicos y humanos: aulas inteligentes y profesores mejor preparados para ayudar a enfrentar al sujeto con las exigencias del siglo XXI. Mark Prensky dice que "es un proceso de docentes, gobierno y estudiantes encaminado a hacer de los sujetos, individuos que se deben enfrentar a un futuro, cada día, más incierto" 11

> Implementar una cultura del E-learning, B-learning para fomentar en los estudiantes la cultura del autoaprendizaje, como una manera de mejorar en conocimiento y aplicabilidad. Se necesitan más laboratorios prácticos; pero también se necesita mucha humanidad para entender que ante todo somos seres humanos e interactuamos con otros seres humanos.

$>$ Si bien la selección de profesores cada día es más exigente en la universidad, se da, a veces, una incongruencia entre el conocimiento de ese docente muy preparado y el enseñar proactivo. De ahí las voces de docentes que saben mucho de teoría y nada de experiencia o de gente con mucha experiencia que no sabe explicar.

$>$ En algún momento se habló, por parte del Ministerio de Educación Nacional de un mecanismo para hacer que el estudiante comience su vida universitaria desde los 18 años. Esto porque un adolescente de 16-17 años quizás no tenga la visión de un adulto de 18-19 años. Se ha hablado, entonces de comenzar estudios primarios a los ocho años de edad; además de

11 Programa Redes. Televisión Española. Emisión $\mathbf{N}^{\circ}$ 75. Eduard Punset entrevista a Mark Prensky. Consultado en http://www.youtube. com/watch?v=xkhKbu_wHiM Junio de 2011. otros mecanismos, entre otros, las calificaciones. Eliminar el porcentaje del $5 \%$ para perdedores, más bien hacer que la escuela sea un proceso que implique no sólo aprobación sino aprendizaje.

\section{Y... Los sueños...}

Retirase de estudiar no siempre entraña fracaso. Para algunos estudiantes significa un "descanso" en el largo proceso educativo y que, de paso, le ayuda a enfrentarlo más adelante con mayor ahínco y entereza. Para otros es un prepararse mientras llega la verdadera prueba en la universidad que desean, mientras que ésta es un "campo de entrenamiento" en el que se aprende a vivir la vida de la educación superior con su falta de disciplina, su libertad de horarios, de cátedra; pero con la suprema responsabilidad de saber hacer bien las cosas so pena de "perder". Y si se pierde no es solo las materias, el sitio en una sociedad que mide los conocimientos, o el sitio en el entorno familiar.

Hay quienes se "quedan en la calle" pues su vida laboral comienza a ofrecerles otras oportunidades más lucrativas y dejan el proceso educativo para un auto aprendizaje que les permita "legalizar" esos conocimientos adquiridos a través de la experiencia.

Tal vez sea por eso que hay estudiantes que consideran la universidad como un lugar en el qué "acreditar" un conocimiento que riñe con la realidad. De ahí que haya alumnos que cuestionan la enseñanza de un docente que no cuenta con la experiencia y cuyo "conocimiento" se refiere a la literatura y a los títulos obtenidos por estudiar y no por practicar.

Ante esa perspectiva, cada día son más las voces que hablan de cambiar paradigmas educativos, de cambiar la forma de enseñar y transformarla en un aprendizaje de todos, donde el estudiante que tiene experiencia debería convertirse en docente de docentes y los docentes 
deberían aprender a ser aprendices para hacer lo que actualmente se busca de ellos: "aprender a preguntar".

En la medida que avanza la tecnología, en la medida que las redes sociales y la Internet se convierten en los centros sociales de la juventud, también comienzan otras voces a "ver" el potencial de éstas en el proceso educativo. Ahora se habla de aprendizajes múltiples, de estudiar desde el celular, de aprovechar la tecnología para convertir un aula de clase concreta con 35 alumnos en un aula virtual con cientos de alumnos que coadyuven a la creación de un conocimiento que cada día es más colectivo. Donde las individualidades van a ser la parte de un todo encaminado a mejorar la raza humana.
Porque, como dice Freire: "Nadie educa a nadie, nadie se educa solo los hombres se educan entre sí mediatizados por el medio".

Cabe preguntarse, pues, si los estudiantes terminarán por desaparecer de las universidades y si los profesores dejarán de serlo. Más bien va a haber un cambio en las maneras de enfrentar las universidades. Los estudiantes entenderán que no sólo es profesor aquel de diga su parlamento (aquel de las hojitas amarillentas); sino que aprenda que ya no detenta el conocimiento sino que sabe dónde encontrarlo, dónde compartirlo y dónde hacerlo posible.

\section{Bibliografía}

Arrieta Vergara Katherine, Díaz Caballero, Antonio y Vergara Hernández Clara Inés. (2009). Deserción Estudiantil en un programa de Odontología en una Universidad Pública de Cartagena. Santa Marta: Revista Duazary (Vol $6 \mathrm{~N}^{\circ}$ 2) Facultad de Ciencias de la Salud. Universidad del Magdalena

Batista Jiménez, Eduardo y Otros. (2005). Mortalidad académica, deserción y cambio de programa en la Facultad de Ingeniería de la Universidad de Antioquia. Medellín: Revista de la facultad de Ingeniería de la Universidad de Antioquia.

Bean, John Paul. (1985). Dropouts and Turnover: The Synthesis and Test of a Causal Model of Student Attrition. Research in Higher Education. 12, 155-187. New York, NY. USA.

Bourdieu, Pierre. (1991). El sentido práctico, Madrid: Editorial Taurus.

Castaño V, Elkin; Vélez Johanna et al. (2003). Determinantes de la Deserción Estudiantil en la Universidad de Antioquia. Medellín: Centro de Investigaciones Económicas CIE - Universidad de Antioquia.

Correa, Carlos Arturo, y Páramo, Gabriel Jaime. (2008). Indicadores de Deserción. Universidad EAFIT. Medellín: Editorial EAFIT.
Cubides, Humberto y otros. (1998). Vivir a toda: Jóvenes, territorios culturales y nuevas sensibilidades. Primera edición. Bogotá: Universidad Central. Siglo del Hombre Editores.

Durkheim, Emili. (1898), El Suicidio. Primera edición. Buenos Aires: Grupo Editorial Tomo.

Garzón, Carlos G et al. (2003). Estudio de la Deserción Estudiantil en la Educación Superior en Colombia. ICFES-UNIVERSIDAD NACIONAL DE COLOMBIA. 2003. [En línea] <www2.unalmed.edu.co/simege> [citado 03/08/2008]

Gaviria, Alejandro. (2002). Los que suben y los que bajan. Educación y movilidad sociales en Colombia. Santafé de Bogotá: Editorial Alfa y Omega.

González González, Miguel Alberto. (2009). Horizontes Humanos. Límites y paisajes. Manizales: Universidad de Manizales.

Guarín Jurado, German. (2010). Módulo de maestría Educación Docencia. Manizales: Universidad de Manizales.

Kaplun Gabriel. (2004). Indisciplinar la universidad. Quito: Universidad Andina Simón Bolívar. 
Lipovetsky, Gilles. (1987). La Era del Vacío, ensayos sobre el individualismo pos-moderno. Barcelona: Anagrama. 2003.

Lopera, Carlos. (2008). Determinantes de la Deserción Universitaria en la Facultad de Economía Universidad del Rosario. Bogotá: Borradores de Investigación Universidad del Rosario \#95, 25.

Maya Guerra, José Ignacio. (2008). Identificación y análisis de factores incidentes en el retiro voluntario de estudiantes de pregrado en el Politécnico Colombiano Jaime Isaza Cadavid en el período 2004-2 a 2006-1. Tesis de Maestría en Ingeniería Administrativa. Universidad Nacional de Colombia, Facultad de Minas. Medellín.

Ministerio de Educación Nacional (2010) Educación Superior. Bogotá. Boletín Informativo \#14 feb. 20p.

Ricoachury, Hernán; Lara Luis. (1984). Los desertores. Universidad Pedagógica Nacional. Cuadernos de Planeación. Bogotá.

Rivera Berrío, Juan Guillermo. (2005). De la deserción a la retención. En: Memoria. Instituto Tecnológico Metropolitano Medellín 38p.

Sánchez Buitrago, Dairo. (2009). Módulo de maestría Educación Docencia. Manizales: Universidad de Manizales.

Spady, William. (1971). Deserción en la Educación Superior. (1970). Chicago: Interchange, 64-85.
Tinto, Vince. (1993). Reflexiones sobre el abandono de los estudios superiores. En: Perfiles Educativos, 62. Madrid.

Tinto, Vince. (1987). Leaving College: Rethinking the Causes and Cures of Student Attrition (2nd ed.). Chicago: The University of Chicago Press.

Universidad de los Andes. (2005). Investigación sobre deserción en las Instituciones de Educación Superior en Colombia. Informe Final. Bogotá: Universidad de los Andes.

Universidad de los Llanos. (2006). Estudio de la deserción estudiantil de los programas de pregrado de la Universidad de los Llanos (1998-2004). Villavicencio: Universidad de los Llanos.

Universidad Nacional de Colombia (2002). Estudio de la deserción Estudiantil en la Educación Superior en Colombia. ICFES. Bogotá: Universidad Nacional..

Universidad Nacional de Colombia. (2007). Cuestión de supervivencia. Graduación, deserción y rezago en la Universidad $\mathrm{Na}$ cional de Colombia. Vicerrectoría General. Bogotá. 266p.

Universidad de Antioquia. (1972). Deserción y mortalidad estudiantil, análisis cuantitativo. Medellín: Universidad de Antioquia, Dirección de Planeación y Desarrollo.

Universidad de Antioquia. (2005). Determinantes de la Deserción Estudiantil en la Universidad de Antioquia. Medellín: Facultad de Ciencias Económicas. 